\title{
The Extended Micro Hot Fusion Scenario
}

Rainer W. Kühne

Tuckermannstr. 35, 38118 Braunschweig, Germany

kuehne70@gmx.de

The cold fusion neutron emissions, neutron bursts, and heat bursts can be explained by the extended micro hot fusion scenario. I describe the model and present the experimental evidence.

During the years 1986 and 1989 three experimental teams independently reported to have discovered cold fusion. The experiments differed strongly from one another, both in the applied methods and the reported results. Hence, the observational results need not necessarily result from one unique physical mechanism. Let us take a brief look at these three types of cold fusion.

Type 1: Mechanically treated LiD and heavy ice samples were reported to have emitted neutron bursts which have lasted for roughly ten minutes [1, 2].

Type 2: Motivated by geophysical observations (anomalous isotope ratios [3, 4]), electrolysis of deuterided metal was performed and reported to have generated low levels of neutrons of $2.5 \mathrm{MeV}$ energy [3]. These emissions appeared a few hours after the start of electrolysis and terminated several hours later [3].

Type 3: Electrolysis of deuterided metals was reported to have emitted high levels of heat appearing days after the start of electrolysis [5-7]. Signals of nuclear fusion (neutrons, gamma rays) were at least 10 orders of magnitude too small to explain the reported heat emissions [57].

The experiments of type 1 were motivated by positive results of fracto-emission experiments and explained by the fracto-fusion model [1, 2]. An analogous "micro hot fusion" scenario was suggested [8] for the explanation of the type 2 experiments.

The micro hot fusion scenario can be described as follows [9, 10].

When hydrogen is absorbed by metals, then it forms bubbles around impurities and lattice defects of the metal. During their growth until a diameter of several micrometers, the bubbles deform the metal lattice and create mechanical stresses. After several hours, the mechanical stresses have become strong enough to create cracks which propagate through the metal lattice. The cracks are formed preferentially between the hydride bubbles and the weaker hydrided metal. Palladium dihydride is a semi-metal. Therefore the different electronegativities of metal and hydrogen generate positively electrically charged hydride bubble surfaces. Hence, the crack sides become electrically charged. Within the cracks of typically one micrometer width and ten to hundred micrometers length there arises an electric field strength of one hundred million volts per centimeter. Within strongly hydrided metals, electrons are bound stronger than hydrogen nuclei. Therefore the electric field within the cracks allows the hydrogen nuclei of the bubbles to accelerate until they reach energies of typically ten kilo-electron-volts.

Within the weaker hydrided metal, the hydrogen nuclei transfer their kinetic energy of several kilo-electron-volts to the metal lattice during a path of one tenth of a micrometer. This energy 
transfer creates hot spots within the hydrided metal with a mean temperature of typically ten thousand degrees Celsius and a pressure of ten billion Pascal. Within the hot spots, the hydrided metal is gaseous. Because of the high internal pressure, the hot spots transfer their heat energy explosively to the surrounding solid hydrided metal, where the explosions generate further cracks. When such a crack collides with the surface of a hydrided bubble, then the electrically charged bubble surface generates a strong electric field within the crack. Again, the electric field accelerates hydrogen nuclei from the bubble until they get several kilo-electron-volts of energy. Hence, a cycle reaction of the creation of cracks, electric fields, kilo-electron-volt hydrogen nuclei, hot spots, and micro-explosions is generated. This cycle reaction might result in the pulverization and even explosion of the entire metal hydride.

If the hydrogen isotope deuterium is used, then this cycle reaction is accompanied by fusion reactions. These fusion reactions occur when the kilo-electron-volt deuterons reach the other crack side and fuse with the non-accelerated deuterons of the weaker hydrided metal. This cycle reaction which includes deuteron fusion reactions is called the "extended micro hot fusion scenario". This scenario was suggested by Roman Sioda and me [9, 11]. It is able to explain the neutron emissions reported by Jones et al. [3, 4], the neutron bursts reported by De Ninno et al. [12, 13] and Menlove et al. [14, 15], the simultaneous heat and neutron bursts reported by Gozzi et al. [16, 17], and the explosions of cold fusion cells reported by Fleischmann et al. [5].

The experimental evidence for the extended micro hot fusion scenario is presented in the table.

Table: Cold Fusion Phenomena which Can Be Explained by Extended Micro Hot Fusion

No. Phenomenon

Explanation

1 | Emission of $2.5 \mathrm{MeV}$ neutrons [3, 4, 18-24] | Deuteron-deuteron fusion d(d, n)3 $\mathrm{He}$

2 | Emission of 3.0 MeV protons [25-27] | Deuteron-deuteron fusion d(d,p)t

3 | Near-surface process for palladium | Crack-formation near palladium | [3, 4, 9, 28] | surface [29]

4 | Deuterium gas emission [30, 31]

| Gas desorption by crack formation [9]

5 | Acoustic emissions simultaneously with | neutron [32, 33] and proton bursts [34]

| Relaxation of metal lattice by

| crack formation [35]

6 | Radio emission simultaneously with proton | bursts [34]

| Formation of high electric fields | within the cracks [35]

7 | Disappearance of neutron emission several | hours after the start of electrolysis | $[3,4,18,19,36]$

| Bubble growth time is between $0.1 \mathrm{sec}$ | and 1 day [37]; fracture time is several | hours [38]

8 Emission of $10 * * 4 \ldots 10 * * 7$ neutrons per $\mathrm{cm}^{* * 3}$ of electrode material (many | experiments where neutrons have been

| Calculation: Refs. [37, 38]

1 


\begin{tabular}{|c|c|c|}
\hline & | detected) & $\mid$ \\
\hline & $\begin{array}{l}\text { Ratio of } 100 \text { emitted neutrons per Joule } \\
\text { liberated }[16,17,30,39,40]\end{array}$ & $\begin{array}{l}\text { Only } 1 \text { of } 10^{* *} 12 \text { of the keV } \\
\text { | deuterons undergoes fusion reactions [11] }\end{array}$ \\
\hline 10 & $\begin{array}{l}\text { | Heat emission from light hydrogen loaded } \\
\text { | cells [41] }\end{array}$ & $\begin{array}{l}\text { | Formation of bubbles, cracks and } \\
\text { | electric fields is independent of the } \\
\text { | hydrogen isotope used }\end{array}$ \\
\hline 11 & $\begin{array}{l}\text { | Emission of keV electrons [42-46], } \\
\text { | positively charged keV ions [43, 47], } \\
\text { | X-rays [48-51], radio-waves [44] and } \\
\text { | electrification [52, 53] from various } \\
\text { | hydrided materials and neutron emission } \\
\text { | [1, 2,54,55] from deuterided materials } \\
\text { | minutes after mechanical treatment }\end{array}$ & $\begin{array}{l}\text { | Fracto-emission by formation of } \\
\text { | strong electric fields with } \\
\mid 10^{* * 7} \ldots 10^{* * 8} \mathrm{~V} / \mathrm{cm} \text { and } \\
\mid 10^{* * 4} \ldots 10^{* * 5} \mathrm{~V}[1,2,50,56] \\
\mid\end{array}$ \\
\hline 12 & | Many non-successful experiments [57-87] & $\begin{array}{l}\text { | Various possible explanations including } \\
\text { | }[9,88-90] \text { : } \\
\text { (a) too low sensitivity of detectors; } \\
\text { (b) electrodes had long pre-loading } \\
\text { times and measurements were } \\
\text { performed only after the termination } \\
\text { of the emissions }\end{array}$ \\
\hline
\end{tabular}

\section{REFERENCES}

1. V. A. Klyuev et al., Sov. Tech. Phys. Lett. 12, 551 (1986).

2. B. V. Derjaguin et al., Colloid. J. USSR 48, 8 (1986).

3. S. E. Jones et al., Nature 338, 737 (1989).

4. S. E. Jones et al., J. Fusion Energy 9, 199 (1990).

5. M. Fleischmann et al., J. Electroanal. Chem. 261, 301 (1989).

6. M. Fleischmann et al., J. Electroanal. Chem. 263, 187 (1989).

7. M. Fleischmann et al., J. Electroanal. Chem. 287, 293 (1990).

8. J. S. Cohen and J. D. Davies, Nature 338, 705 (1989).

9. R. W. Kühne, Fusion Technol. 25, 198 (1994).

10. R. W. Kühne, Fusion Technol. 37, 265 (2000).

11. R. W. Kühne and R. E. Sioda, Fusion Technology 27, 187 (1995).

12. A. De Ninno et al., Nuovo Cimento A 101, 841 (1989).

13. A. De Ninno et al., Europhysics Letters 9, 221 (1989).

14. H. O. Menlove et al., J. Fusion Energy 9, 215 (1990).

15. H. O. Menlove et al., J. Fusion Energy 9, 495 (1990).

16. D. Gozzi et al., Nuovo Cim. A 103, 143 (1990).

17. D. Gozzi et al., SIF Conf. Proc. 24, 241 (1990).

18. A. Bertin et al., Nuovo Cim. A 101, 997 (1989).

19. A. Bertin et al., J. Fusion Energy 9, 209 (1990).

20. K. L. Wolf et al., J. Fusion Energy 9, 105 (1990).

21. K. L. Wolf et al., AIP Conf. Proc. 228, 341 (1991).

22. T. Bressani et al., Nuovo Cim. A 104, 1413 (1991).

23. E. Botta et al., Nuovo Cim. A 105, 1663 (1992). 
24. M. Bittner et al., Fusion Technol. 23, 346 (1993).

25. R. Taniguchi et al., Jap. J. Appl. Phys. 28, L2021 (1989).

26. S. E. Jones et al., AIP Conf. Proc. 228, 397 (1991).

27. G. F. Cerofolini et al., Nuovo Cim. A 105, 741 (1992).

28. N. Wada and K. Nishizawa, Jap. J. Appl. Phys. 28, L2017 (1989).

29. P. B. Price, Nature 343, 542 (1990).

30. E. Yamaguchi and T. Nishioka, Jap. J. Appl. Phys. 29, L666 (1990).

31. Y. Arata and Y. C. Zhang, Proc. Jpn. Acad. B 66, 1 (1990).

32. P. I. Golubnichii et al., AIP Conf. Proc. 228, 151 (1991).

33 P. I. Golubnichii et al., JETP Lett. 53, 122 (1991).

34. P. I. Golubnichii et al., AIP Conf. Proc. 228, 146 (1991).

35. P. I. Golubnichii et al., Sov. Phys. Dokl. 34, 628 (1989).

36. B. Emmoth et al., SIF Conf. Proc. 24, 79 (1990).

37. S. E. Segre et al., Europhys. Lett. 11, 201 (1990).

38. V. A. Tsarev, Sov. Phys. Usp. 33, 881 (1990).

39. D. Gozzi et al., J. Fusion Energy 9, 241 (1990).

40. A. G. Lipson et al., Sov. Tech. Phys. Lett. 18, 673 (1992).

41. E. Yamaguchi and T. Nishioka, AIP Conf. Proc. 228, 354 (1991).

42. V. V. Karassev et al., Dokl. Akad. Nauk 88, 777 (1953).

43. J. Wolbrandt et al., Phys. St. Sol. A 27, 53 (1975).

44. J. T. Dickinson et al., J. Mater. Sci. 16, 2897 (1981).

45. J. Mathison et al. J. Appl. Phys. 65, 1923 (1989).

46. A. G. Lipson et al., Sov. Tech. Phys. Lett. 15, 783 (1989).

47. J. T. Dickinson et al., J. Mater. Res. 5, 109 (1990).

48. V. V. Lymar et al., IX-All Union Conf. Acoustics (Moscow, Nauka, 1977), p. 65.

49. T. M. Belyaeva et al., Sov. Tech. Phys. Lett. 10, 341 (1984).

50. V. I. Berkov et al., Sov. Phys. Dokl. 32, 381 (1987).

51. V. A. Klyuev et al., Dokl. Akad. Nauk 279, 415 (1984).

52. M. I. Kornfeld, J. Phys. D 11, 1295 (1978).

53. Yu. I. Golovin et al., Sov. Phys. Sol. St. 27, 671 (1985).

54. M. A. Yaroslavskii, Sov. Phys. Dokl. 34, 637 (1989).

55. M. A. Yaroslavskii, Sov. Phys. Dokl. 34, 648 (1989).

56. B. V. Derjaguin et al., Sov. Phys. Dokl. 19, 208 (1974).

57. M. Gai et al., Nature 340, 29 (1989).

58. E. Kashy et al., Phys. Rev. C 40, 1 (1989).

59. K. D. Schilling et al., Z. Phys. A 336, 1 (1990).

60. R. D. Armstrong et al., Electrochimica Acta 34, 1319 (1989).

61. R. D. Armstrong et al., J. Electroanal. Chem. 272, 293 (1989).

62. N. S. Lewis et al., Nature 340, 525 (1989).

63. D. E. Williams et al., Nature 342, 375 (1989).

64. M. H. Salamon et al., Nature 344, 401 (1990).

65. M. M. Broer et al., Phys. Rev. C 40, 1559 (1989).

66. M. R. Deakin et al., Phys. Rev. C 40, 1851 (1989).

67. K. E. Rehm et al., Phys. Rev. C 41, 45 (1990).

68. J. R. Southon et al., Phys. Rev. C 41, 1899 (1990).

69. J. F. Ziegler et al., Phys. Rev. Lett. 62, 2929 (1989).

70. P. B. Price et al., Phys. Rev. Lett. 63, 1926 (1989).

71. J. P. Briand et al., Phys. Lett. A 145, 187 (1990).

72. R. Aleksan et al., Phys. Lett. B 234, 389 (1990).

73. G. M. Miskelly et al., Science 246, 793 (1989).

74. D. Alber et al., Z. Phys. A 333, 319 (1989). 
75. S. Blagus et al., Z. Phys. A 333, 321 (1989).

76. A. Baurichter et al., Z. Phys. B 76, 1 (1989).

77. G. Schrieder et al., Z. Phys. B 76, 141 (1989).

78. D. A. Roberts et al., Phys. Rev. C 42, 1809 (1990).

79. J. J. G. Durocher et al., Can. J. Phys. 67, 624 (1989).

80. R. Behrisch et al., Nucl. Fusion 29, 1187 (1989).

81. J. Roth et al., Nucl. Fusion 30, 441 (1990).

82. G. P. Chambers et al., Phys. Rev. B 41, 5388 (1990).

83. G. M. McCracken et al., J. Phys. D 23, 469 (1990).

84. F. Botter et al., Phys. Lett. B 232, 536 (1989).

85. V. Eberhard et al., Z. Phys. A 334, 357 (1989).

86. B. Balke et al., Phys. Rev. C 42, 30 (1990).

87. I. F. Silvera and F. Moshary, Phys. Rev. B 42, 9143 (1990).

88. R. W. Kühne, Phys. Lett. A 155, 467 (1991).

89. R. W. Kühne, Phys. Lett. A 159, 208 (1991).

90. R. W. Kühne, Magazin2000plus 232, 24 (2006) ( = arxiv: physics/0604119). 